\title{
El valor público, característica fundamental en la nueva gestión pública
}

\section{Public value, a fundamental characteristic in the new public management}

\author{
Walter Zegarra ${ }^{1}$ iD*., Delicia Olano ${ }^{2}$ (D)., Marco Lluncor ${ }^{1}$ iD, y Adolfo Cacho $^{3}$ iD.
}

\begin{abstract}
RESUMEN
El artículo tuvo por objetivo analizar el valor público como característica fundamental en la nueva gestión pública. El tipo de investigación fue cuantitativa, la muestra seleccionada fue de 375 funcionarios, aplicándose el muestreo no probabilístico cuyo nivel de confianza fue del $95 \%$, la técnica empleada fue la encuesta y el instrumento el cuestionario con escala tipo Lickert, el método utilizado para la recogida de la información fue en forma presencial y online. Se determinó que los organismos locales defienden improvisaciones de servicios públicos sin una instauración del valor público. Las improvisaciones que demandan instituir vínculos con los habitantes se observan insuficientes y con predisposición a la baja. Por último, se concluye que la generación de valor público se expone en un 32,5\% por la correlación inmediata con el logro de resultados desafiantes, que a la vez depende elocuentemente del desarrollo de competencias, dirección centrada en el control de gestión, asignación de recompensas y una cultura de mejora continua.
\end{abstract}

Palabras clave: Valor público, gobernanza, globalización, nueva gestión pública.

\begin{abstract}
The aim of the article was to analyse public value as a fundamental characteristic of new public management. The type of research was quantitative, the sample selected was 375 civil servants, applying non-probabilistic sampling with a confidence level of $95 \%$, the technique used was the survey and the instrument was the questionnaire with a Lickert scale, the method used to collect the information was face-to-face and online. It was determined that local bodies advocate improvisations of public services without the establishment of public value. Improvisations that require the establishment of links with the inhabitants are found to be inadequate and with a predisposition to decline. Finally, it is concluded that the generation of public value is $32.5 \%$ exposed by the immediate correlation with the achievement of challenging results, which in turn depends eloquently on competence development, leadership focused on management control, allocation of rewards and a culture of continuous improvement.
\end{abstract}

Keywords: Public value, governance, globalization, new public management.

DOI: https://doi.org/10.37787/pakamuros-unj.v9i1.160

Recibido: 22/09/2020. Aceptado:15/11/2020

* Autor para correspondencia

1. Doctorando Universidad Cesar Vallejo-Chiclayo, Perú. Email: waltereloy_19@ hotmail.com

2. Doctorando Universidad Cesar Vallejo-Chiclayo, Perú. Email: delly_Ola@live.com

3. Doctorando Universidad Cesar Vallejo-Chiclayo, Perú. Email: marco.lluncor@gmail.com

4. Doctorando Universidad Cesar Vallejo-Chiclayo, Perú. Email: acachorevilla@gmail.com 


\section{INTRODUCCIÓN}

El concepto de valor público, se percibió desde antes de la segunda guerra mundial, se confunde muchas veces las funciones del sector público ligado con el privado (Blaug, 2006). Investigadores exponen como los gobiernos de turnos de esa época implementaron acciones en favor de la población que fracasaron por la acción incorrecta de las personas que estuvieron al frente de estos escenarios (Fernández, 2018). Similar caso se presentó a nivel de Sudamérica, donde más prevaleció los actos de corrupción, intereses políticos y la poca capacidad de las personas responsables de los destinos nacionales, regionales y locales (Kolhberg, 1982). El mal accionar de estos personajes llevó a realizar un acto de reflexión de cómo se generando el valor público en la sociedad, lamentable indicar que a pesar de la crisis sanitaria actual a raíz de la pandemia COVID-19, los procesos de adquisición de insumos para combatir dicha situación, se demostró la poca capacidad de los funcionarios, administrativos, entre otros.

La atención de la ayuda humanitaria trasladada a la población no contribuyó el efecto positivo, ya que los primeros beneficiados fueron personas con solvencia económica, dejando de lado a los más humildes, trayendo abajo la relación estado-ciudadanos al no proteger su derecho como poblador, no prestar un eficiente servicio y no satisfacer su necesidad, contrario a las funciones del sector privado que busca valor público a través de la rentabilidad, para todo estado el valor es la satisfacción y la garantía del derecho público (Chilet, 2019).

El estado busca modernizarse, innovarse, prevaleciendo el servicio a brindar para la población, observándose variadas formas de innovación que pretendan resolver los problemas que afectan a la misma, siendo relativamente insuficientes, implicando que éstas no siempre responden a las variadas confusiones de los problemas públicos actuales (ENAP, 2017).

Todo esto nos lleva a preguntarnos como el estado aporta en la generación del valor público en sus funcionarios, servidores públicos y población misma; problema que no es efectiva, eficiente, sustentable o simplemente mejor que las soluciones existentes y que desde décadas pasadas, los resultados no fueron los óptimos ya que no va de la mano con la nueva gestión pública.

Sobre la base de lo mencionado el objetivo del presente artículo, es analizar el valor público como característica fundamental en la nueva gestión pública, con los cambios y la modernización de las tecnologías de la información y comunicación.

\section{MATERIALES Y MÉTODOS}

De la exhaustiva revisión al material seleccionado, en la metodología prevaleció el enfoque cualitativo y cuantitativo, puesto que, para el primer caso, se realizó una revisión concreta de la información sobre el 
valor público en los lugares seleccionados donde se dirigieron los estudios, análisis fundamentado de la forma como los gobiernos implementaron sus programas en beneficio de los ciudadanos. Destacando las opiniones de diversos autores de renombre que produjeron obras de importancia teórica, incluso aportes empíricos que fortalecieron los modelos de administración pública conocidos como la tradicional y la nueva gestión pública (Zurriggen \& Gonzáles, 2015). Para el caso de los estudios cuantitativos, se basó en la aplicación de encuestas con escala tipo Lickert, destacando dimensiones como el liderazgo por resultados, dirección basada en el control de gestión, asignación de recompensas, desarrollo de competencias, logro de resultados desafiantes, cultura de cambio, aprendizaje mejora contínua centrada en el valor de la organización y la creación del valor público.

El análisis de fiabilidad individual de los indicadores, se tomó como base el análisis factorial en su evaluación de viabilidad, la cuantificación se realizó con el índice Kaiser-Meyer-Oklin (KMO), siendo superior a 0.50 y la prueba de esfericidad de Barlett $(\mathrm{PEB})$ debiendo ser significativa $(\mathrm{p}<0.05)$. En este proceso se utilizó la plataforma SPSS v.20.

\section{RESULTADOS}

La formación de valor público se acredita en indicadores de medición que aludan a formas de medición sobre cómo se aprovechan los recursos públicos y cómo su aplicación produce mejores externalidades positivas a la sociedad civil, a través del método de extracción de máxima verisimilitud (Tabla 1).

Tabla 1. Índice KMO y PEB

\begin{tabular}{lcc}
\hline \multicolumn{1}{c}{ Constructo } & KMO & Significancia de Barlett \\
\hline L.C.R. $\left(^{*}\right)$ & 0,789 & 0,000 \\
D.B.C.G. $(* *)$ & 0,850 & 0,000 \\
D.C. $(* * *)$ & 0,855 & 0,000 \\
A.R. $(+)$ & 0,826 & 0,000 \\
L.O.G.R. $(++)$ & 0,688 & 0,000 \\
C.U.L.M.C. $(+++)$ & 0,743 & 0,000 \\
V.P. $\left(^{*+}\right)$ & 0,635 & 0,000
\end{tabular}

Fuente: Cabana, Cortés, Contreras \& Vargas (2020); KMO= Kaiser-Meyer-Oklin; PEB= Prueba de Esfericidad de Barlett Reseña:

(*) Liderazgo centrado en resultados

(**) Dirección basada en control de gestión

(***) Desarrollo de competencias

(+) Asignación de recompensas

(++) Logros de resultados desafiantes 
$(+++)$ Cultura de mejora continua

(*+) Valor Público

Para admitir un indicador como componente de un constructo, se contempló las cargas factoriales con equivalencias óptimas a 0.4 sugerido por Hair, Black, Babin y Anderson (2014) y para conseguir rigor en los fundamentos se excluyeron aquellas cargas factoriales con valores inferiores a 0.5 (Cabana, Cortés, Contreras, \& Vargas, 2020)

En la Tabla 2, Los resultados de las cargas factoriales de las variables estudiadas, no cumplen con el mínimo establecido; por tanto, fueron desechados (LCR6, DBCG1, DBCG2, DBCG3, DBCG5, DBCG7, DBCG8, DBCG9, DBCG12, DBCG16, LOGR1, LOGR2, LOGR3, LOGR8 y CULMC3).

Tabla 2. Cargas factoriales

\begin{tabular}{|c|c|c|}
\hline Constructo & Indicador & Carga factorial $(\chi)$ \\
\hline \multirow{6}{*}{$\begin{array}{l}\text { Liderazgo centrado en } \\
\text { resultados }\end{array}$} & LCR1 & 0,866 \\
\hline & LCR2 & 0,756 \\
\hline & LCR3 & 0,857 \\
\hline & LCR4 & 0,860 \\
\hline & LCR5 & 0,869 \\
\hline & LCR6 & $-0,056$ \\
\hline \multirow{17}{*}{$\begin{array}{l}\text { Dirección basada en control de } \\
\text { gestión }\end{array}$} & DBCG1 & 0,213 \\
\hline & DBCG2 & 0,211 \\
\hline & DBCG3 & 0,341 \\
\hline & DBCG4 & 0,508 \\
\hline & DBCG5 & 0,307 \\
\hline & DBCG6 & 0,523 \\
\hline & DBCG7 & 0,127 \\
\hline & DBCG8 & 0,483 \\
\hline & DBCG9 & 0,317 \\
\hline & DBCG10 & 0,804 \\
\hline & DBCG11 & 0,836 \\
\hline & DBCG12 & 0,193 \\
\hline & DBCG13 & 0,860 \\
\hline & DBCG14 & 0,798 \\
\hline & DBCG15 & 0,812 \\
\hline & DBCG16 & 0,218 \\
\hline & DBCG17 & 0,586 \\
\hline \multirow[t]{3}{*}{ Valor público } & VP1 & 0,916 \\
\hline & VP2 & 0,833 \\
\hline & VP3 & 0,798 \\
\hline \multirow[t]{5}{*}{ Desarrollo de competencias } & DC1 & 0,851 \\
\hline & $\mathrm{DC} 2$ & 0,835 \\
\hline & DC3 & 0,776 \\
\hline & DC4 & 0,849 \\
\hline & DC5 & 0,783 \\
\hline \multirow[t]{5}{*}{ Asignación de recompensas } & AR1 & 0,920 \\
\hline & AR2 & 0,836 \\
\hline & AR3 & 0,795 \\
\hline & AR4 & 0,811 \\
\hline & AR5 & 0,682 \\
\hline \multirow{3}{*}{$\begin{array}{l}\text { Logro de } \\
\text { desafiantes }\end{array}$} & LOGR1 & $-0,153$ \\
\hline & LOGR2 & $-0,030$ \\
\hline & LOGR3 & 0,083 \\
\hline
\end{tabular}




\begin{tabular}{lcc}
\hline & LOGR4 & 0,840 \\
& LOGR5 & 0,719 \\
& LOGR6 & 0,846 \\
& LOGR7 & 0,855 \\
& LOGR8 & $-0,002$ \\
Cultura de mejora continua & CULMC1 & 0,867 \\
& CULMC2 & 0,567 \\
& CULMC3 & 0,394 \\
& CULMC4 & 0,819 \\
& CULMC5 & 0,852 \\
\hline
\end{tabular}

Fuente: Cabana, Cortés, Contreras \& Vargas (2020); Leyenda: Liderazgo centrado en resultados (LCR); Dirección basada en control de gestión (DBCG); Desarrollo de competencias (DC); Asignación de recompensas (AR); Logro de resultados desafiantes (LOGR); Cultura de mejora continua (CULMC); Valor público (VP).

En lo que respecta al análisis de fiabilidad y validez del constructo, se examinó por intermedio del alfa de cronbach y el coeficiente de fiabilidad compuesta (IFC). En la Tabla 3, se descubre un equilibrio interno en los 7 constructos considerados, resaltando los niveles confiados de 0,7 . Por otro lado, se observa que el IFC resalta los valores recomendados, por lo que se comprueba la armonía interna de las variables del instrumento de evaluación.

Tabla 3. Índice de fiabilidad interna, fiabilidad compuesta y validez convergente

\begin{tabular}{lccc}
\hline \multicolumn{1}{c}{ Constructo } & $\begin{array}{c}\text { Alfa de } \\
\text { Cronbach }\end{array}$ & IFC & $\begin{array}{c}\text { Validez } \\
\text { convergente } \\
\text { (AVE) }\end{array}$ \\
\hline $\begin{array}{l}\text { Liderazgo centrado en resultados (LCR) } \\
\text { Dirección basada en control de gestión }\end{array}$ & 0,897 & 0,897 & 0,710 \\
(DBCG) & & 0,876 & 0,541 \\
Desarrollo de competencias (DC) & 0,875 & 0,873 & 0,671 \\
Asignación de recompensas (AR) & 0,864 & 0,865 & 0,660 \\
Logro de resultados desafiantes (LOGR) & 0,834 & 0,873 & 0,667 \\
Cultura de mejora continua (CULMC) & 0,797 & 0,826 & 0,617 \\
Valor público (VP) & 0,807 & 0,850 & 0,723 \\
\hline
\end{tabular}

Fuente: Cabana, Cortés, Contreras \& Vargas (2020)

La validez convergente se logra comprobar por medio de la varianza extraída media (AVE) de los constructos. El coeficiente AVE supera el valor mínimo recomendado de 0,5 en todos los valores alcanzados y demuestra su significancia al 95\%, confirmando que las variables explican más del 50\% de la varianza de sus constructos, por lo tanto, existe validez convergente (Cabana, 2020) (Tabla 4).

Tabla 4. Validez discriminante

\begin{tabular}{cccccccc}
\hline Constructo & AR & CULMC & DC & DBCG & LCR & LOGR & VP \\
\hline AR & 0,812 & & & & & & \\
CULMC & 0,055 & 0,786 & & & & \\
DC & 0,545 & 0,032 & 0,810 & & & \\
DBCG & 0,168 & 0,230 & 0,114 & 0,843 & & \\
LCR & 0,205 & 0,241 & 0,077 & 0,159 & 0,735 & & \\
\hline
\end{tabular}




\begin{tabular}{cccccccc}
\hline LOGR & 0,163 & 0,639 & 0,345 & 0,375 & 0,225 & 0,817 & \\
VP & 0,065 & 0,709 & 0,042 & 0,141 & 0,182 & 0,325 & 0,850 \\
\hline
\end{tabular}

Fuente: Cabana, Cortés, Contreras \& Vargas (2020): Liderazgo centrado en resultados (LCR); Dirección basada en control de gestión (DBCG); Desarrollo de competencias (DC); Asignación de recompensas (AR); Logro de resultados desafiantes (LOGR); Cultura de mejora continua (CULMC); Valor público (VP)

Se logró visualizar la evaluación de las relaciones causales propuesto en el modelo, a través de la varianza explicativa $\left(\mathrm{R}^{2}\right)$. El valor $\mathrm{R}^{2}$ para el valor público es de 0,475 , entendiéndose que el $47.5 \%$ de la varianza del actual constructo es aplicado en el modelo, y existe una proximidad del $48 \%$ de la varianza del valor público viene definitiva para el beneficio de efectos desafiantes. La Tabla 5, muestra que el índice de ajuste $(\mathrm{GoF})$ es 0,404 lo que demuestra que hay un ajuste en el modelo de medida y en el modelo estructural, cumpliéndose de esta manera con el criterio empírico respecto a que la medida de bondad de ajuste ostente una variación de 0 y 1 , a significativo valor; en consecuencia, mejor será el índice.

Tabla 5. Varianza explicada e índice de bondad de ajuste

\begin{tabular}{lcc}
\hline \multicolumn{1}{c}{ Constructo } & $\begin{array}{c}\text { Validez convergente } \\
\text { (AVE) }\end{array}$ & $\mathbf{R}^{\mathbf{2}}$ \\
\hline Liderazgo centrado en resultados (LCR) & 0,710 & \\
Dirección basada en control de gestión (DBCG) & 0,541 & 0,125 \\
Desarrollo de competencias (DC) & 0,671 & 0,112 \\
Asignación de recompensas (AR) & 0,660 & 0,103 \\
Logro de resultados desafiantes (LOGR) & 0,667 & 0,519 \\
Cultura de mejora continua (CULMC) & 0,617 & 0,163 \\
Valor público (VP) & 0,723 & 0,475 \\
\hline
\end{tabular}

Fuente: Cabana, Cortés, Contreras \& Vargas (2020)

Para el contraste de hipótesis en relación con los coeficientes PATH o pesos de regresión estandarizados $(\beta)$, los valores deseables deberán estar por encima de 0,3 siendo 0,2 un valor mínimo aceptable. (Tabla 6). Las hipótesis $\mathrm{H} 3, \mathrm{H} 4, \mathrm{H} 5$ y H9 no superaron el valor de 0,2 que se considera como el minúsculo valor admitido, las subsiguientes relaciones modelan con la regla de quedar sobre el pequeño valor consentido.

Tabla 6. Contraste de hipótesis

\begin{tabular}{cllcc}
\hline Hipótesis & \multicolumn{2}{c}{ Relaciones } & Coeficiente PATH & Contraste \\
\hline H1 & LCD & CULMC & 0,248 & ACEPTA \\
H2 & LCR & DBCG & 0,275 & ACEPTA \\
H3 & DBCG & DC & 0,114 & RECHAZA \\
H4 & DBCG & AR & 0,168 & RECHAZA \\
H5 & DBCG & CULMC & 0,035 & RECHAZA \\
H6 & DBCG & LOGR & 0,265 & ACEPTA \\
\hline
\end{tabular}




\begin{tabular}{|c|c|c|c|}
\hline $\mathrm{H} 7$ & CULMC & 0,680 & ACEPTA \\
\hline H8 & LOGR & 0,222 & ACEPTA \\
\hline H9 & AR LOGR & 0,126 & RECHAZA \\
\hline H10 & LOGR VP & 0,325 & ACEPTA \\
\hline
\end{tabular}

Fuente: Cabana, Cortés, Contreras \& Vargas (2020)

\section{DISCUSIÓN}

Según el análisis desarrollado, se puede visualizar claramente la carencia de valor público en las instituciones (Rodríguez, 2017). Pensando que el Liderazgo acertado en resultados posee una predominio concreto y válido, fundamentado en el control de gestión y esta última asociada con el Desarrollo de Competencias y la Cultura de Mejora Continua, intervienen directa y efectivamente con significancia estadística, en el Logro de Resultados Desafiantes de este organismo público, en un 26,5\%, 22,2\% y $68 \%$ respectivamente, a su vez influye de carácter inmediata y significativa en el Valor Público, en un 32,5\%. Lo descrito guarda mucha relación con lo vertido por (Chilet, 2019) quién describe claramente el concepto de valor público.

Una gestión sistémica de estas variables originará la instauración de valor público, fundando parcialmente un "ecosistema innovador", donde la cooperación entre diferentes representantes (Comunidad, Ministerio y Gobierno), conceptúen un encadenamiento que beneficia el incremento y sostenibilidad de distintos servicios y pilotos de trabajo, donde el control de gestión como instrumento que gravita a la dirección de las instituciones, a través del informe formado habitualmente, permitirá a los decisores estatales saber e instruirse de modo proactivo, como lo que ocurre en cada instante, en los métodos calificados críticos para la entidad. Similar situación se presenta con lo manifestado por Aguilar \& Monforte (2018) en el caso del área metropolitana de Monterrey. De esta forma, los servidores públicos poseerán la persuasión que los esquemas de acción y tácticas de perfeccionamiento país, como sus efectos, igualmente son útiles para ellos, en consecuencia, continuarán empoderando y producirán periódicamente, extendiendo los logros de resultados desafiantes que impactan claramente en el Valor público generado. Otro punto importante, es que los gobiernos locales desde la perspectiva del valor público, las innovaciones relacionales componen un indicador de utilidad en los gobiernos municipales por abastecer servicios públicos atendiendo a las perspectivas, provechos y horizontes de complacencia de los habitantes. Enuncia además el nivel de innovación a partir del piloto fundado en la "oferta" de servicios públicos a otro respaldado en la "demanda", en que la decisión, la gestión y el liderazgo del perfeccionamiento y edificación de bienes públicos descansan en los dependientes privados, comunitarios y de la sociedad civil (López, 2016). 
En términos de creación de valor público, las decisiones internamente del rubro "participación ciudadana" benefician una excelente formación de acciones del gobierno y el sector público con las insuficiencias de los habitantes, originando un conjunto de servicios, acoplado y flexible hacia quienes va encaminada. Por otro lado, los proyectos afines a la "transparencia y rendición de cuentas" consienten comunicar y exponer a los ciudadanos las gestiones ejecutadas por el gobierno municipal, es decir, favorecen a la evaluación del accionar público (López, 2016). Es indudable que los gobiernos municipales poseen un extenso camino que atravesar y afrontan trascendentales dificultades en su camino hacia la cimentación de valor público. Entre los más significativos se logran sugerir: la prevención al riesgo, las obligaciones burócratas, la escasa cabida de gestión, los espacios de corto plazo, presupuestos limitados, corrupción, provechos económicos, omisión de liderazgo y carencia de incentivos.

Existen ciertas limitaciones conocidas por aquellos investigadores del tema tratado, puesto que el estado está al servicio de la población, los programas creados en muchos de los casos no llega a los ciudadanos de escasos recursos económicos, específicamente los pobres, creando desigualdades entre las personas que reciben estos beneficios y los que son olvidados, en mucho de los casos el liderazgo del funcionario que está al frente de una entidad pública genera inconvenientes al momento de trasladar los mismos (Aguilar, 2018).

La falta de un programa sostenible y la transparencia pública genera desconfianza en los gobernantes de turno, seguido de la poca o casi nula experiencia en gestión pública que opaca su administración, muy por el contrario, limita tener una aceptable ejecución del gasto en obras de envergadura que ayude a los pueblos de su jurisdicción salir de la pobreza y pobreza extrema (Tuñez, 2018). Sin embargo; según lo analizado, esta acción se puede revertir, accionando en favor del poblador prestándole un servicio eficiente, comunicando y transparentando su labor, evitando los actos impropios desafiantes que impactan claramente en el Valor público (López, 2016).

\section{CONCLUSIONES}

Para crear valor público, a todos los gobiernos de turno les corresponde suministrar servicios públicos acorde a sus posibilidades, con beneficios y niveles de agrado para el poblador, es preciso conocer las insuficiencias de los protagonistas favorecidos para conseguir valorar los horizontes de agrado con la eficacia de los productos y servicios compensados que influya directa y positivamente en el valor público generado por los servicios dependientes de dichos entes públicos y según la nueva gestión pública, a todas las personas se les debe tratar como clientes, tal como lo realizan la mayoría de empresas con sus usuarios, existe una oferta y una demanda por satisfacer si hablamos de servicios públicos, con los 
recursos existentes, se facilitan esos servicios y prestaciones; caso contrario, se definirán otros con limitaciones, siempre al servicio de la población.

\section{REFERENCIAS BIBLIOGRÁFICAS}

Aguilar, I., \& Monforte, G. (2018). Public water services, public value and sustainability: The Monterrey Metropolitan area case. Gestión y Política Pública, 149-179.

Blaug, R. (2006). Heritge, Democracy and Public Value. Eniversity of Westminster.

Cabana, R., Cortés, F., Contreras, F., \& Vargas, V. (2020). Influence of management control on public value generated in services dependent on the ministry of economy, development and tourism, Chile. Información Tecnológica, 103-116.

Chilet, J. (2019). Desarrollo moral y valor público. Saber Servir, 34-44.

ENAP. (2017). Estado y Valor Público. Programa Formativo para la mejora de servicios públicos. Lima: SERVIR.

Fernández, J. (2018). Public Value, Governance and Third Way. Convergencia, 175-193.

Hair, J., Black, W., Babin, B., \& Anderson, R. (2014). Multivariate data analysus. EE.UU.: Pearson.

Kolhberg, L. (1982). The Philosophy of Moral Development. EE.UU.: British Journal of Psichology.

López, D. (2016). Public value and the integration agreements in Colombia. Administración y Desarrollo, 72-88.

López, J. (2016). Innovation and creation of public value in mexican local governments. Iberoamericana de las Ciencias Sociales y Humanísticas, 1-17.

Moore, M. (2006). Creando valor público a través de asociaciones público privadas. Revista CLAD Reforma y Democracia, 1-22.

Rodríguez, M. (2017). Public value tests as a result of governance adaptation to public service media. Latina, 1682-1698.

Tuñez, M. (2018). Europen public broadcasters interaction and social media policy to public value management. Prisma Social, 61-90.

Zurriggen, C., \& Gonzáles, M. (2015). Co-creating public value. Challengers for Latin America. CTS, 143-171. 